\title{
Internet of Things: Wildlife Conservation and its Challenges
}

\author{
Ratnesh Kumar Choudhary \\ Professor, Department of Computer Science \& Engineering, S. B. Jain Institute of Technology, Management \& Research, \\ Nagpur, Maharashtra, India \\ E-mail: ratneshkumarchoudhary@gmail.com
}

\begin{abstract}
Right now there are more connected "Things" than there are people on this planet. Early last year, it is predicted that Internet of things (IOT) would have at least 8.4 billion connected "Things" in use rising to more than 21 billion by 2021. Today major percentage of wildlife is vulnerable to extinction. This is caused due to the destruction of forest areas for farming, logging and construction of new cities and towns. Many wild animals have been killed due to road accidents and speeding vehicles passes through the wildlife protected area. Animals crossing boundaries have led to be killed. The impacts of roads include habitat loss, habitat fragmentation and habitat degradation that affect wildlife and its habitats both directly and indirectly especially on larger mammals [6]. Recent studies into the extent of illegal logging estimate that illegal logging accounts for $\mathbf{5 0 - 9 0}$ per cent of the volume of all forestry in key producer tropical countries and 15-30 per cent globally [1]. Protection of wildlife has become an important now a days, because of human activities such as hunting of wild animals and cutting of trees which causes major threat to the wildlife. Recent advancements in IoT technology and sensor technology have the potential to wildlife conservation. Physical barriers are not suitable for animals because they may harm the animal physically. This can be prevented by using tracking of animal movement and keep them under a logical barrier. This paper focuses on implementation of animal monitoring and challenges of wildlife conservation. This paper also shows natural challenges and technical challenges while implementing animal tracking and health monitoring system.
\end{abstract}

Keywords: Wildlife conservation, animal tracking, Health monitoring, Internet of Things, challenges in animal conservation, wireless sensor network.

\section{INTRODUCTION}

For the past few years, utilization of Internet of Things (IoT) have grown steadily. Wildlife monitoring is one of the trending applications of IoT technologies, where several heterogeneous sensors are deployed to monitor the activities of wild animals dwelling in a remote and geographically large habitat. It is known that wild animals depict similar physiological activities as a herd [5]. Internet of things (IoT) \& Wireless sensor networks (WSNs) together have been widely used for wildlife monitoring and tracking. Generally, different functional devices working as sensors are used to report animal data to the base station. In this paper, we focus on the animal monitoring and tracking in large wild areas. In an unknown large area, it is difficult and sometimes infeasible to find wildlife animals and attach wearable tracking devices to them. In this case, data is reported. The animals which are under observation is attached a collar kit which will consist of a microprocessor, many sensors such as heartbeat sensor, temperature sensor, pulse rate sensor, respiratory sensor etc. These sensors is attached to the microprocessor for collecting information from various sensors, these date will then be transmitted to the ZIG BEE receiver for further processing. The collar kit is also having a GPS which helps in tracking of animal movement. All these tracking \& health related data is given to microcontroller for monitoring purpose. Implementation of this IoT based wildlife health monitoring system is not easy as it faces many challenges. Few challenges are natural challenges, and few are technical challenges.

This paper discussion has been done on the detailed architecture and implementation of IoT based animal tracking and health monitoring system. Also, discussion on many general and technical challenge faced during the implementation of the system have been presented. The challenges of wildlife conservation have been considered based on Africa's forest and other major forest in the world. In the II section literature survey has been presented. III section of this paper focuses on wild animal tracking \& health monitoring by using technology such as IoT, Sensors and other technology. IV section of the paper presents general challenges which we have faced during implementation of wildlife tracking \& health monitoring system. In $\mathrm{V}$ section of the paper focuses on technical challenges faced during implementation of wildlife tracking \& health monitoring system. Section VI present result and discussion based on the output of the model. Lastly in section VII conclusion has been given.

\section{LITERATURE SURVEY}

Mr. S. Syed Husain [1] proposed a system in which, the design uses three sensors tilt sensor (to detect the inclination of tree when its being cut), temperature sensor(to detect forest fires),sound sensor (for effective detection of illegal logging i.e. even the sounds generated while axing the tree are also sensed) Data generated from these sensors is continuously monitored. This system can monitor real-time related parameters, e.g., temperature, relative humidity, and send the data immediately to the computer of the monitoring. The collected data will be analyzed and managed by the computer. Compared with the normal meteorological information and basic forest resource data, 
the system can make a quick assessment of a potential fire danger. With respect to the sensors, their output devices are activated through relay switch. For RF transmitter used in animal body to track and identify to communicate and interface in RF receiver in microcontroller and sound sensor a buzzer is activated and for temperature sensor a water pump is activated.

Luís Nóbrega's [2] explored the SheepIT cloud platform that receives animal sensor streamed data, performs data analysis (e.g. rule management), allows farm managers to access animal information and trigger alarms in real-time when certain situations are detected (e.g. panic attacks, attacks from predators, abnormal number of infractions, etc.). The paper also describes some preliminary results of an animal posture monitoring use case, for which several machine learning algorithms were tested. The Sheep IT project aims at offering an IoT-based solution for monitoring, controlling, and managing herds of sheep weeding vineyards. To reach such goal, the system comprises several distinct blocks (and respective interactions), each of them being responsible for specific tasks. Among these tasks, the data gathering, data aggregation, data processing and data representation may be highlighted. Such local infrastructure is in turn interconnected through a wide-band connection (e.g 3G, 4G, LTE) to a cloud platform.

Jun $\mathrm{Xu}$ [3] focuses on the animal monitoring and tracking in large wild areas. Sensor identifies animal's appearance by different types of inputs such as smell, sound and image. With deployed sensors, the system gets animal appearance information in wildlife areas. Jun $\mathrm{Xu}$, divide the whole observation area into small virtual grids. Then based on this grid structure network, Jun Xu designed the system model by utilizing a UAV and letting the UAV to explore and learn these" hot" small virtual grids. By treating each grid as a cluster of sensor nodes, data collection by the UAV is reduced to visiting the cluster head of each grid. The Markov decision process (MDP) model is used to do path planning for the UAV as each grid represents a state of MDP. We solve the MDP model using Q-learning algorithm by letting UAV to receive rewards from grids when animal data is reported. The goal of our path planning approach is collecting animal data efficiently and then predicting future animal movements. The UAV is self-learning and can dynamically plan and adapt according to environment changes.

Eyuel D. Ayele [5] proposed a WMS network architecture by utilizing LPWAN and opportunistic mobile networks (OMN). The results and analysis were presented by considering LoRa as an LPWAN technology and BLE as an enabler for opportunistic network. This type of architecture can find use in wildlife scenarios where LoRa can act as collection stations, and collared BLE sensor nodes as regular sources that generate and concatenate data in the network. The author proposed a new IoT network architecture for wildlife monitoring system along with preliminary prototype deployment of animal tracker. Also, he investigates the performance of opportunistic BLE network with existing data dissemination protocols in terms of delivery ratio and average latency.

\section{WILD ANIMAL TRACKING AND HEALTH MONITORING}

The given model is indented to be helpful for all animal saver and doctors who can closely monitor animal health activities. And in case, of animal saver they can even search wild animals if they are missing.

The figure 1 consists of four important sensors called, heart rate sensor, temperature sensor, pulse rate sensor and respiratory sensor. A Global Positioning System is deployed to track the movement of an animal in case if the animal is missing or lost. The wireless communication technology used would be ZigBee. ZigBee has very low power consumption range of 10-3000 meters and it can support up to 64000 devices having 50 meters [4].



Fig.1 Transmitter with sensors

In figure 2, the data from the ZigBee transceiver receives the data from the microcontroller embedded in the system and sends the data to a PC using UART protocol. The data may be raw and needs editing. However, a software system will be developed in next phase to analyze the data.



Fig.2 Zig bee receiver \& monitoring unit 
In figure 3, the data values from the hardware will be given to a website to be processed. The website/webpage will have all the 5 data values along with analysis of it. And once the data is analyzed, we can monitor based on some threshold (temperature of a dog should be between 37.939.9 degree) if there is any threat to animal health. For example, a sudden increase in temperature after monitoring for hours can suggest that the animal may be suffering from fever. And suggestion of medicines can be given. If there is a critical condition, then the regular doctor may be alerted. To develop and match the system with current trends, the system would be available on any device using IOT. IOT will bridge the gap between devices and will also enable users and owners to freely check upon their pets or animals from anywhere. Since the data receiving from the device is live and continuous, the data would be stored in cloud.

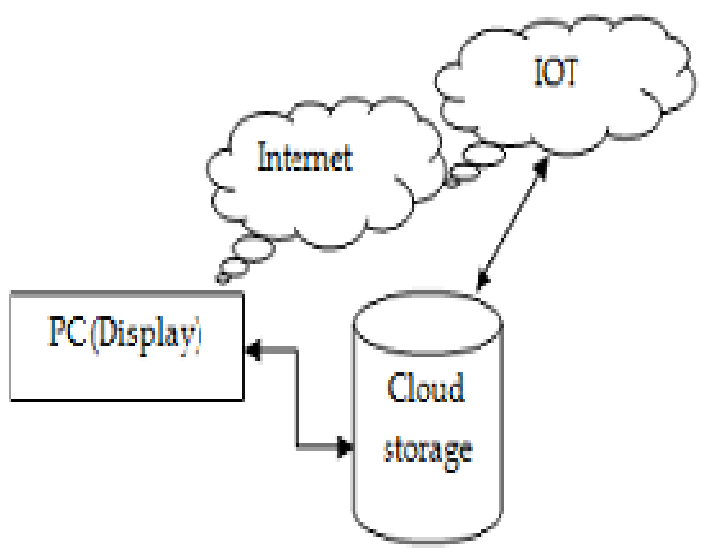

Fig.3 Data storage on cloud using IOT

\section{A. Temperature Sensor Module}

Wild animal has a core body temperature (CBT) which gives an indication of their body temperature. Any deviation from their usual temperature is a cause for abnormality. For example, a wild cow has body temperature between 38.0 to 39.3 degree Celsius, to measure this, we use LM35 temperature sensor. The LM35 is rated to operate over a $-55^{\circ}$ to $+150^{\circ} \mathrm{C}$ temperature range [4].

\section{B. Heart Rate Sensor Module}

Heartbeat sensor is designed to give digital output of heat beat when a finger is placed on it. When the heartbeat detector is working, the beat LED flashes in unison with each heartbeat. This digital output can be connected to microcontroller directly to measure the beats per minute (BPM) rate. It works on the principle of light modulation by blood flow through finger at each pulse.

\section{Pulse Rate Sensor Module}

The sensor clips onto a fingertip or earlobe and with some jumper cables. It also includes an open-source monitoring app that graphs your pulse in real time. It essentially combines a simple optical heart rate sensor with amplification and noise cancellation circuitry making it fast and easy to get reliable pulse readings. It calculates the pulse rate over a minute.

\section{Respiratory Sensor Module}

The Respiration Rate Sensor includes a Relative Pressure Sensor and inflatable belt, which wraps around the chest of animal. As the animal breathes, respiration rate and pressure changes can be displayed in a graph on a computer. When the belt is connected to the Relative Pressure Sensor, the Pressure sensor measures the pressure in the bladder of the belt as the lungs expand and contract. The pressure of the lungs and chest cavity expanding causes pressure against the bladder of the belt, but the pressure measured is not the actual pressure change in the lungs.

\section{E. Global Positioning System}

The Global Positioning System (GPS) is a satellite-based navigation system that can be used to locate positions anywhere on earth. Designed and operated by the U.S. Department of Defense, it consists of satellites, control and monitor stations, and receivers. GPS receivers take information transmitted from the satellites and uses triangulation to calculate an animal's exact location.

\section{F. Zig Bee Transceiver}

ZigBee is an enabling technology providing low data rate, low battery consumption with low cost based on the standard IEEE 802.15.4. It is contributing to wireless personal area network and wireless sensor network. ZigBee have capability to make a large network, it can support up to 64000 devices having distance 50 meters [4]. It needs very low power.

\section{INTERNET OF THINGS}

The Internet of Things (IoT) is the network of physical objects-devices, vehicles, buildings, and other items embedded with electronics, software, sensors, and network connectivity-that enables these objects to collect and exchange data. IoT allows objects to be sensed and controlled remotely across existing network infrastructure, creating opportunities for more direct integration between the physical world and computer-based systems, and resulting in improved efficiency, accuracy, and economic benefit. The given wildlife tracking and health monitoring system was implemented in real mode with all the four mentioned sensors. The received values from sensors was transferred over the wireless ZigBee communication channel through UART protocol and is shown in both the LCD as well as the computer through the putty software. As a result, it is proved that the integration of GPS and sensors in the same microcontroller is possible without any complications and, the data retrieved is accurate and there is no interference in the data. [4] 


\section{GENERAL CHALLENGES IN WILDLIFE CONSERVATION}

\section{A. Deforestation \& Climate Change}

Africa is experiencing water stress, droughts, and an increase in arid lands due to climate change-effects that are being exacerbated by deforestation. Because forests act as critical carbon sinks while also serving as water catchments, AWF is employing a couple of strategies to keep forests in Africa intact. In Kenya, agriculture, logging, and settlement have reduced the Mau Forest complex, a water catchment for east Africa to a quarter of its original size. AWF has been planting and protecting seedling and implementing alternative live hood. As of late 2015 AWF has rehabilitated about 337.5 hectare of the forest here [12]. Meanwhile in south Kenya, north central Tanzania and Democratic Republic of the Congo, AWF has been using reducing Emissions from Deforestation and forest Degradation as a strategy to incentive communities can claim carbon credits for protecting a forest and sell those credits on the voluntary carbon market.

\section{B. Resource Extraction}

Too, often, government in Africa have given mining or oil concession inside, or perilously close to, national park boundaries. Such operation can damage the eco system and open protected areas to illegal bush meat hunting. In November 2014, AWF join with 7 other NGO's to call for a no-go policy around World Heritage Site Support Network, an NGO consortium; AWF is also building the capacity of wildlife authorities in African World Heritage Sites [12]. These includes simian mountains national park in Ethiopia and the Dja Faunal Reserve in Cameroon. By providing rangers with the technology and training to accurately record and analyze ecological data from patrols, we are ensuring that wild life authorities are well trained to protect these valuable areas from poaching-which typically escalates when mining is conducted nearby-and identify and report direct environmental impact related to resource extraction.

\section{Bush meat Hunting}

For many in rural Africa, bush meat hunting provides a much-needed source of protein for rural communities. Rangers at Murchison Falls have picked up almost 5 tons of snares in the past two years [12]. Through Urgent Response Fund, AWF provides funding support to local groups like Game Rangers International to address Bus Meat hunting. In the Congo landscape, where the Bus Meet market is also alive and well, AWF is tackling the problem in slightly different ways. AWF conducted a six month survey in 2014 to determine how much, and what types, of illegal Bus Meat were being sold in four major local markets, then conducted a workshop to sensitize both local officials and those engaged in the trade.

\section{Human- Wild Life Conflict}

Human wildlife conflict is a big problem in many part of Africa. In Kilimanzaro landscape in Kenya, for example, only three elephants died in 2015 from poachers engaged in a wildlife trade- but 24 were killed due to human elephant conflict. The Historic Amboseli- Chyule corridor that is use by elephants in shrinking quickly due to human settlement and agriculture. As a result, elephants end up frequently trading on- or outright raiding- crops. People then spear and kill elephants in retaliation. As a first line of defense against marauding elephants, community scouts figure prominently in efforts to minimize human animal conflict. From Kenya to Uganda, AWF- supported scouts used chili bricks made from chili powder and cow dung and then lit to produce chili infuse smoke, beehive fences, and noise makers such as Vuvuzelas to turn elephants from village farmlands [12].

\section{E. Population Growth, Land-use change}

For better or for worse, many of this conservation issues are interlinked-and many are the result of large forces. Human population growth, for example, has resulted in people settling in once open areas, exacerbating conflict with wildlife. Population growth is also leading to rampant land conservation and land use change-for agriculture, settlement, and infrastructure development. As a result, again greater conflict with wildlife and less natural habitat for wildlife have been observed [12]. Ultimately, it may come down to land use planning, to ensure sufficient space for human activity and for wildlife. Such plan allows for appropriate development while limiting sprawl.

\section{TECHNICAL CHALLENGES IN WILDLIFE CONSERVATION}

As estimated $15 \%$ of animals are found using microchips, identification collars for other pet tracking IOT devices, and that figure is growing year over year [13]. But while these devices are already in high demand among animal saver, getting these devices on to the market can be complicated. IoT based animal tracking device manufacturers need to build a wearable that is not overly large or bulky. This means that the tracking technology used in a animal collar, needs to be as portable as possible without sacrificing tracking accuracy over long distances. If collar is too heavy it will be impractical for smaller animal's breeds, since it would strain on the animal's neck, resulting in health issues and general discomfort. Yet manufactures who make a device that is too flimsy could risk damage to the product during everyday use. An animal tracking IoT device needs to be durable enough to withstand the elements and an animal rough and tumble lifestyle- without adding significant weight or bulk to the product.

Moreover, the tracking software and hardware that these devices utilize must be fast, reliable, long lasting, and accurate enough for animal trackers to track the lost animals within just a few yards. By contrast, tracking collars that use 
subpar components may only be able to determine that an animal is somewhere in the neighborhood, which does not give the animal saver enough information to zero in on the animals' precise location. In addition, a reliable animal tracking IoT device also need to have enough battery life to withstand days, month or even years of use. We will need to ensure that our device battery does not die before we have the chance to find a lost pet.

The data gathered from sensor is largely superficiallocation, movement, temperature, respiration etc. and offers limited value in terms of health. The parameters associated with monitoring animal health conditions may evade detection [11]. Also, tracking how much an animal's eats, sleep and play does not really explain why they quit using her litter box. Animal monitoring is difficult in remote large-scale wildlife areas due to the dangerous environment and the uncertainty of animal movement patterns.

\section{RESULT AND DISCUSSION}

Figure 4 and 5 shows the tracking location of Animal in the wildlife reserves or national parks. This project utilizes a GPS modem and a GSM modem for this purpose. Forest officer or Government authority person will get these SMS containing Longitude and Latitude.

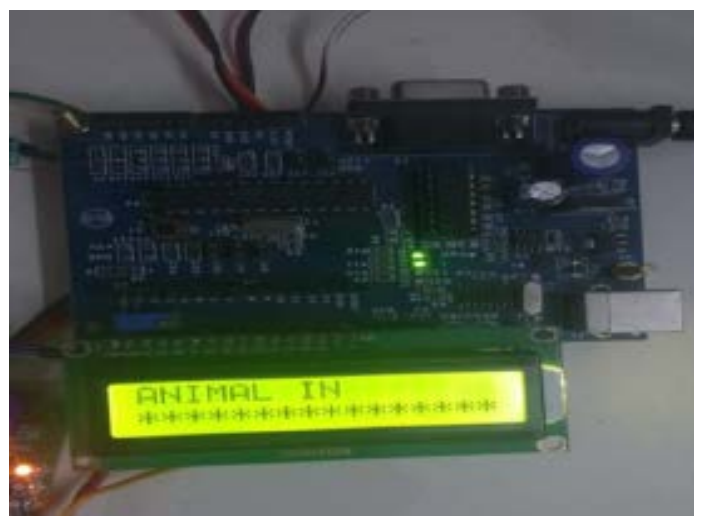

Fig.4 Animal location tracking (animal in)

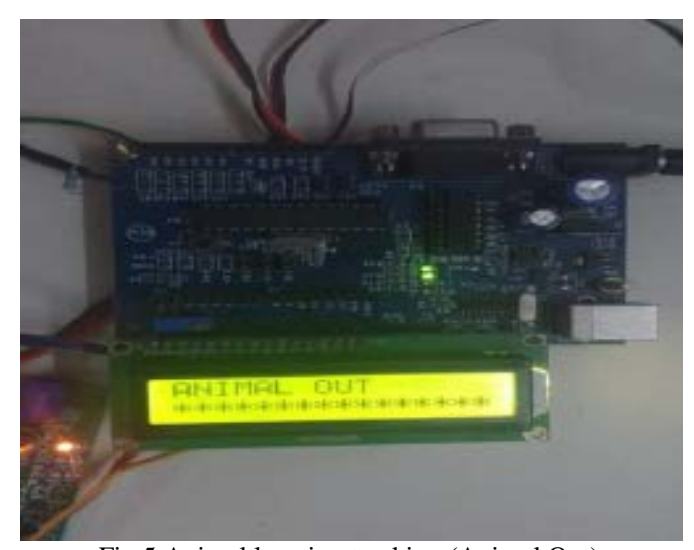

Fig.5 Animal location tracking (Animal Out)

A temperature sensor measures the hotness of an object. Fig 6 shows the temperature rises whenever the voltage increases. The sensor records any voltage drop between the transistor base and emitter.

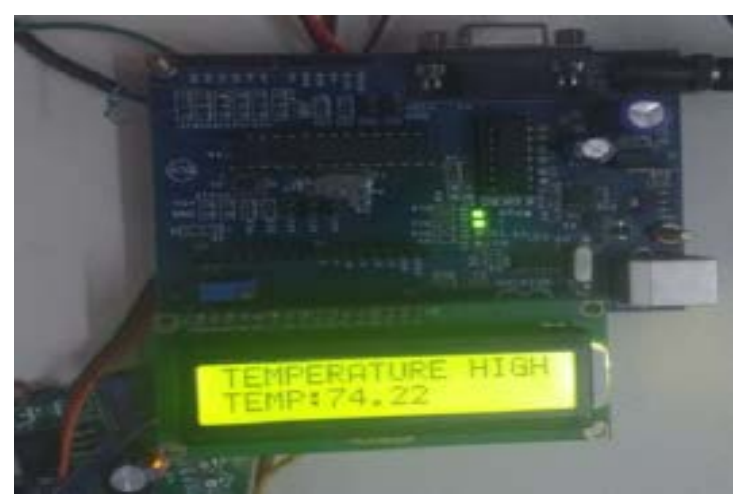

Fig.6 Temperature Measurement

\section{CONCLUSION}

The proposed system can track animal location within the predefined range. It can detect animal movement. Also, it can measure the body temperature of the animal. Apart from that it can also read heart rate and respiratory information. Based on this information, the animal saver can plan their activity to help the animal if they are in dangerous or abnormal condition as far as health of animal is concern. Also, it can help the animal saver to know location of animal and monitor them. The proposed system will provide safety and security to wildlife and help to increase their numbers which are on extinction. Animal tracking system is useful for tracking and monitoring of animals. So, they can be protected from illegal hunting, killing, or capturing of wild animals. Embedded system based this project provides accurate health information and location of the animal. IoT and Sensor networks give accurate result for a short distance and for shorter period. There are lots of general and technical challenges which the system must take into consideration for more accurate result. Few technical challenges such as battery life, weight of the kit can be easily solved but there are few general challenges which cannot be removed fully but can be reduced to greater extends by policy makers, people living in the forest and government support.

\section{REFERENCES}

[1] Mr.S.Syed Husain, "Design and Implementation of Forest Monitoring System using IOT”, IJARCCE, Vol. 8, No. 5, May 2019

[2] Luís Nóbrega, “Animal monitoring based on IoT technologies", IEEE, 2018

[3] Jun $\mathrm{Xu}$, “Internet of Things Applications: Animal Monitoring with Unmanned Aerial Vehicle”, Arvix, Oct-2016

[4] P.Keertana, "IOT based animal health monitoring \& Tracking system using Zig Bee.” , IJRTI-2017, Vol. 2, No. 4, ISSN: 2456-3315

[5] Eyuel D. Ayele, "Towards A New Opportunistic IoT Network Architecture for Wildlife Monitoring System”, Pervasive Systems Research Group, University of Twente, Enschede, the Netherlands”

[6] SK Supreeth , "IoT - Wildlife Monitoring, Virtual Fencing with Deforestation Notifications”, IRJET, Vol. 6,No. 2, Feb 2019 
[7] Andy Rosales Elias, "Where's The Bear?- Automating Wildlife Image Processing Using IoT and Edge Cloud Systems", IoTDI 2017, Rosales Elias et al., Pi.sburgh, PA USA, April 2017

[8] S.Sheela, "Low Cost Alert System for Monitoring the Wildlife from Entering the Human Populated Areas Using IOT Devices", IJIRSET, Vol. 5, No.10, May 2016

[9] Dr. Kirti Wankhede, "Animal Tracking and Caring using RFID and IOT”, IOSR Journal of Computer Engineering (IOSR-JCE) e-ISSN: 2278-0661,p-ISSN: 2278-8727, pp. 24-27.
[10] B S Sudha, "Forest Monitoring System Using Wireless Sensor Network", IJASRE, Vol.4, No. 4 April - 2018

[11] Thomas Overbay,"The future of the internet of Animals”, Global AgInvesting, Nov. 2018.

[12] Mayu Mishina, "Beyond Wildlife Trafficking: other conservation challenge"s, African Wildlife foundation, April 2016.

[13] Benoit Tournier, "Iot tracking technology taes the stress out of pet ownership" at IoT blog at sierra wireless. 